\title{
Stepwise Evolution and Exceptional Conservation of ORF1a/b Overlap in Coronaviruses
}

\author{
Han Mei, ${ }^{1}$ Sergei Kosakovsky Pond, ${ }^{2}$ and Anton Nekrutenko (D)*,1 \\ 'Department of Biochemistry and Molecular Biology, The Pennsylvania State University, University Park, PA, USA \\ ${ }^{2}$ Department of Biology, Institute for Genomics and Evolutionary Medicine, Temple University, Philadelphia, PA, USA \\ *Corresponding author: E-mail: aun1@psu.edu. \\ Associate Editor: Aya Takahashi
}

\begin{abstract}
The programmed frameshift element (PFE) rerouting translation from ORF1a to ORF1 $b$ is essential for the propagation of coronaviruses. The combination of genomic features that make up PFE-the overlap between the two reading frames, a slippery sequence, as well as an ensemble of complex secondary structure elements-places severe constraints on this region as most possible nucleotide substitution may disrupt one or more of these elements. The vast amount of SARSCoV-2 sequencing data generated within the past year provides an opportunity to assess the evolutionary dynamics of PFE in great detail. Here, we performed a comparative analysis of all available coronaviral genomic data available to date. We show that the overlap between ORF1a and ORF1b evolved as a set of discrete 7, 16, 22, 25, and 31 nucleotide stretches with a well-defined phylogenetic specificity. We further examined sequencing data from over 1,500,000 complete genomes and 55,000 raw read data sets to demonstrate exceptional conservation and detect signatures of selection within the PFE region.
\end{abstract}

Key words: SARS-COV-2, frameshift, conservation.

Coronaviruses have large $26-32 \mathrm{kbp}$ positive-strand RNA genomes. The initial $2 / 3$ of the genome is occupied by an open reading frame (ORF) ORF1ab encoding nonstructural proteins essential for the coronaviral life cycle. As the designation "ab" suggests, it contains two reading frames with the $3^{\prime}$-end of ORF $1 a$ overlapping with the $5^{\prime}$-terminus of ORF $1 b$. ORF $1 b$ is in -1 phase relative to ORF1a and translated via the -1 programmed ribosomal frameshifting controlled by the PFE. As ORF1b encodes crucial components of coronavirus transcription/replication machinery, including the RNAdependent RNA polymerase (RdRp), disrupting PFE abolishes viral replication completely (Brierley 1995; Plant et al. 2010; Sola et al. 2015; Kelly et al. 2020). PFE consists of three consecutive elements: 1) an attenuator loop, 2) the "NNN WWW H" slippery heptamer, and 3) a pseudoknot structure (Kelly et al. 2020; Huston et al. 2021). The sequence and structural conformation of these elements determine the efficiency of the frameshift event, which ranges from $15 \%$ to $30 \%$ in SARS-CoV and SARS-CoV-2 (Baranov et al. 2005; Kelly et al. 2020). Because disruption of PFE arrests viral replication, it is a promising therapeutic target. As a result, a number of recent studies have scrutinized its characteristics (reviewed in Rangan et al. (2021)) revealing a fluid secondary structure (Iserman et al. 2020; Ziv et al. 2020; Huston et al. 2021). In addition to secondary structures, PFE harbors the overlap between ORF1a and ORF1b. It is defined as the stretch of sequence from " $\mathrm{H}$ " in the slippery heptamer to the stop codon of ORF1a. The position of the ORF1a stop codon determines overlap length. For example, in SARSCoV-2, it is 16 bp, while in mouse hepatitis virus (MHV) it is $22 \mathrm{nt}$ (Plant et al. 2010).

Our group has been interested in the evolutionary dynamics of overlapping coding regions (Nekrutenko et al. 2005; Chung et al. 2007; Szklarczyk et al. 2007). The vast amount of newly generated sequence and functional data-a result of the current SARS-CoV-2/COVID-19 pandemic-provides an opportunity to re-examine our current knowledge. The length of the ORF1a and ORF1b overlap is phylogenetically conserved. It evolved in a stepwise manner, where the changes in the overlap length are results of the loss of ORF1a stop codons leading to ORF1a extension, and the acquisition of insertions and deletions causing early stops of ORF1a.

Distance-based methods had shown that the $\delta$-coronavirus genus was an early split-off lineage compared to $\alpha-\beta$-, and $\gamma$-coronavirus (fig. 1). Comparisons of the RdRp, $3 \mathrm{CL}^{\text {pro }}$, HEL, $M$, and $N$ proteins suggested that $\gamma$ - was more closely related to $\delta$-coronavirus, while $\alpha$ - and $\beta$-coronavirus cluster together forming a distant clade (de Groot et al. 2012; Lau et al. 2012; Woo et al. 2012; Coronaviridae Study Group of the International Committee on Taxonomy of Viruses 2020). However, comparing the $S$ protein trees, $\alpha$ - and $\delta$-coronavirus 


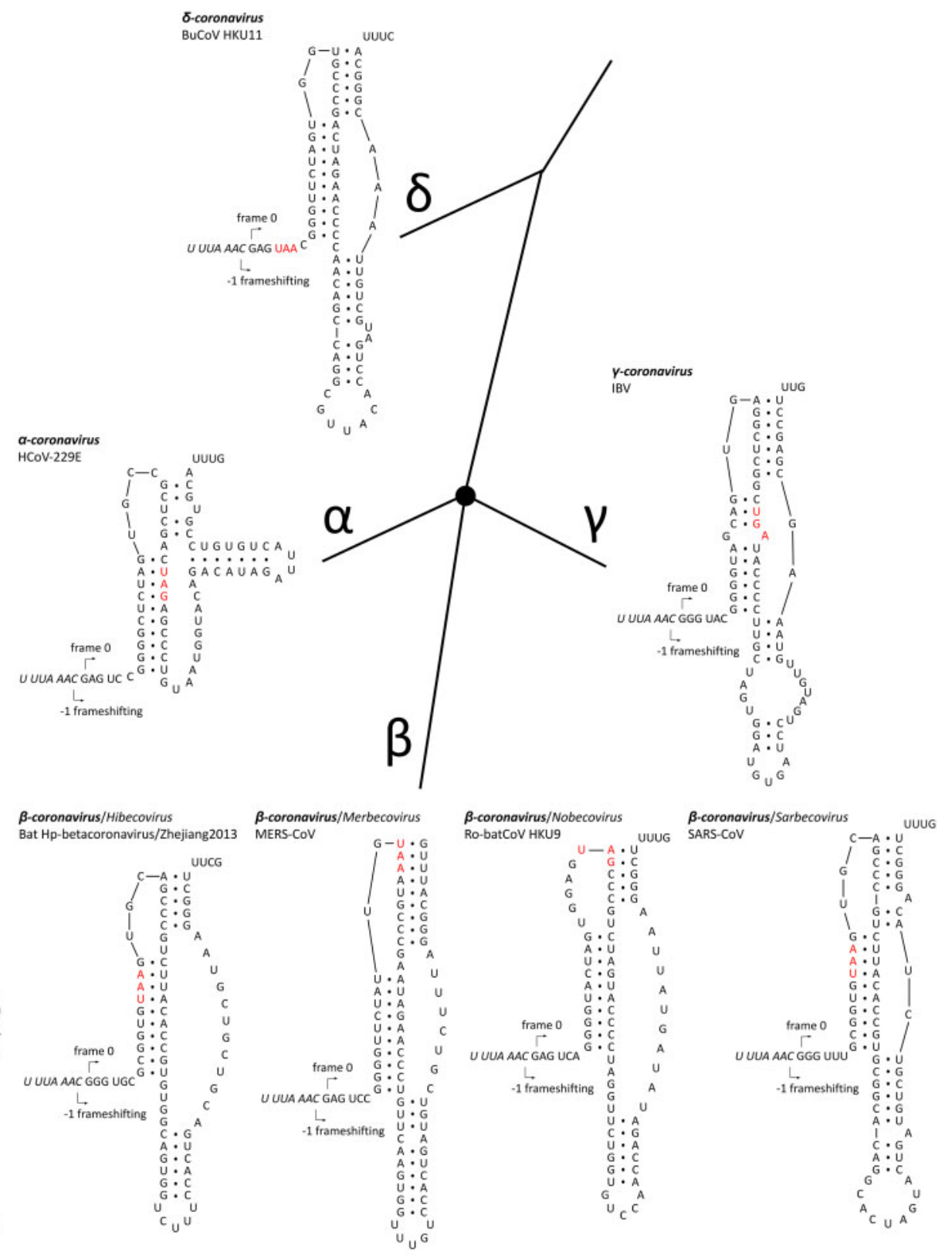

FIG. 1. PFE slippery sites and pseudoknot structures in coronaviruses. The slippery site "UUUAAAC" is shown in italic. The ORF1a stop codon is shown in red. ORF1 $b$ frame starts from the " $C$ " in the slippery site. $\alpha$-, $\beta$-, and $\gamma$-coronavirus were plotted as splitting from one common node (black filled circle), with no phylogenetic order shown. The pseudoknot structures of SARS-CoV and MHV are redrawn based on Plant et al. (2010). The pseudoknot structures of HCoV-229E and IBV are redrawn based on Plant et al. (2005). HCoV-229E, human coronavirus 229E; NC_002645.1. MHV, mouse hepatitis virus; NC_001846.1. Bat Hp- $\beta$-coronavirus/Zhejiang2013, NC_025217.1. MERS-CoV, NC_019843.3. Ro-batCoV HKU9, rousettus bat coronavirus HKU9, NC_009021.1. SARS-CoV, NC_004718.3. IBV, infectious bronchitis virus, NC_001451.1. BuCoV HKU11, bulbul coronavirus HKU11, NC_011547.1.

share a higher amino acid identity, while $\beta$ - and $\gamma$-coronavirus cluster together (Lau et al. 2012). Due to this, we initially assumed that $\alpha, \beta$, and $\gamma$ formed an unresolved trifurcation (fig. 1). To assess all possible configurations within this region, we surveyed all genomic sequences of family Coronaviridae available from the National Center for Biotechnology Information (NCBl; see Materials and Methods section). The distribution of overlap lengths among 4,904 coronaviral genomes (supplementary table S1, Supplementary Material online) is shown in supplementary fig. 1, Supplementary Material online. There are five distinct overlap length groups $(7,16,22,25$, and $31 \mathrm{nt})$ with clear taxonomic specificity.

We then compared the first 15 amino acids of ORF $1 b$ in all 4,904 entries (fig. 2). The amino acid sequences are highly conserved: positions $1(\mathrm{R}), 2(\mathrm{~V}), 4(\mathrm{G}), 7(\mathrm{~S}), 11-13$ (ARL), and $15(P)$ are almost invariable and highly redundant. Next, we compared the underlying nucleotide sequences of the PFE region (fig. 3). This suggests the following potential series of evolutionary events. $\delta$-Coronavirus with $7 \mathrm{nt}$ overlap most likely represents the ancestral state. Comparing coronaviruses with $7 \mathrm{nt}$ ( $\delta$-coronavirus) and $31 \mathrm{nt}(\alpha$-and $\gamma$-coronavirus) in 


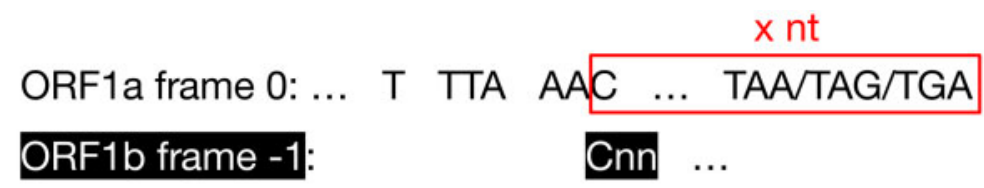

Translation of ORF1b frame -1 , starting from $\mathrm{Cnn}(\mathrm{R})$ :

$\delta$-coronavirus

$\mathrm{x}=7 \mathrm{nt}$

a-coronavirus \& $\gamma$-coronavirus

$\mathrm{x}=31 \mathrm{nt}$

$\beta$-coronavirus/Nobecovirus

$\mathrm{x}=25 \mathrm{nt}$

$\beta$-coronavirus/Embecovirus \&

$\beta$-coronavirus/Merbecovirus

$\mathrm{x}=22 \mathrm{nt}$

$\beta$-coronavirus/Hibecovirus \&

$\beta$-coronavirus/Sarbecovirus

$\mathrm{x}=16 \mathrm{nt}$
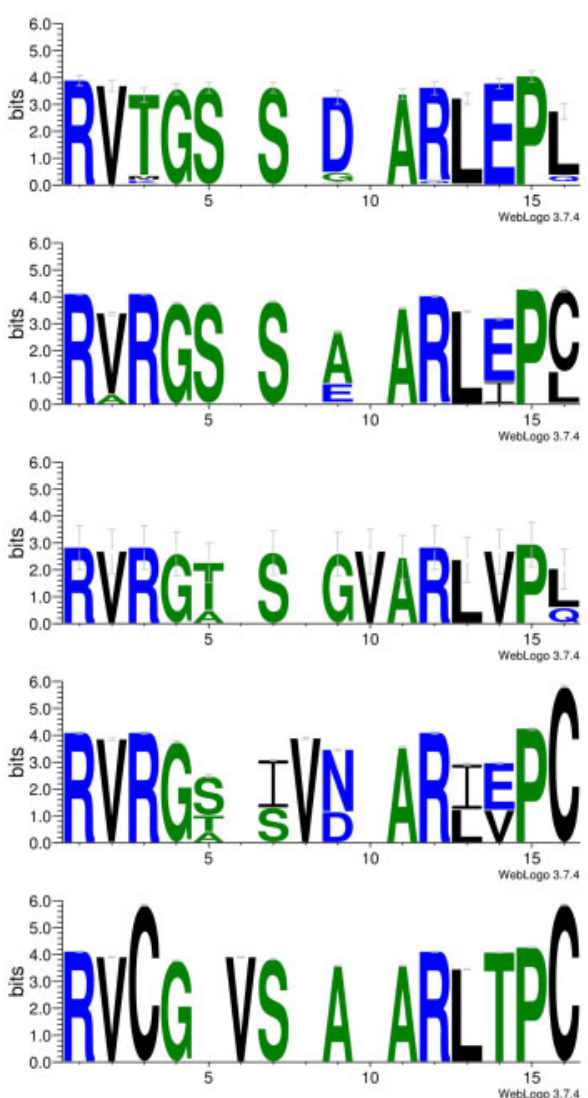

Fig. 2. Amino acid alignment of the first 13-14 amino acids in coronaviruses with different lengths in the overlap region. For each genus/subgenus shown, all coronavirus entries belonging to it were used to generate the consensus amino acid sequences. Gaps are included to maintain alignment.

the overlap, the stop codon which defines a $7 \mathrm{nt}$ overlap is abolished at positions 5-7, through substitutions, which extends ORF1a to the next available stop codon at positions 38-40. This extension results in a new overlap with $31 \mathrm{nt}$ in length (fig. 3A). Comparing coronaviruses with $31 \mathrm{nt}(\alpha$-and $\gamma$-coronavirus) and $25 \mathrm{nt}$ ( $\beta$-coronavirus/Nobecovirus) overlaps reveals a "GTA" insertion at positions 28-30. "TA" from the "GTA" together with the following " $G$ " forms a new stop codon leading to a $31 \rightarrow 25 \mathrm{nt}$ shortening of the overlap. In a Nobecovirus with a $25 \mathrm{nt}$ overlap, the $31 \mathrm{nt}$ overlap stop codon (at positions 38-40) is still observable (fig. 3B). Further comparison of coronaviruses with $31 \mathrm{nt}(\alpha$ - and $\gamma$-coronavirus) and $22 \mathrm{nt}$ ( $\beta$-coronavirus/Embecovirus and Merbecovirus) overlaps revealed a "GTA" insertion as well, but at positions 22-24. "TA" at positions 23-24 and the following " $A$ " or " $G$ " at position 25 constitute a new stop codon. In the 22 nt overlap, substitutions have been observed at the original stop codon (at positions 38-40) from $31 \mathrm{nt}$ overlap coronaviruses; more specifically, " $\mathrm{C}$ " appears at position 39 (fig. 3C). Finally, we compared coronaviruses with 31 and
$16 \mathrm{nt}$ length in the overlap. The same "GTA" insertion footprint was found, at positions 16-18 ahead of the two "GTA" insertions in $31 \rightarrow 25 \mathrm{nt}$ and $31 \rightarrow 22 \mathrm{nt}$ events. "TA" at positions 17-18 and the following " $A$ " at position 19 form the stop codon in the $16 \mathrm{nt}$ overlap coronaviruses. In addition, deletions at positions 13-15 were observed (fig. 3D). These deletions are referred as "TCT"-like, since "TCT" are the dominant nucleotides observed at positions 13-15 in the 7 and $31 \mathrm{nt}$ overlap coronaviruses. At positions 38-40, the ancestral stop codon in the $31 \mathrm{nt}$ overlap coronaviruses cannot be seen, since the nucleotide at position 39 is invariably represented by " $T$ " (fig. 3D). The variable position of the stop codon likely has an implication to the frameshift efficiency in these taxa as was shown by Bhatt et al. (2021). These authors demonstrated that extension of the distance between the slippery heptamer and the stop codon of 0 -frame decreases frameshifting frequency: an increase in the distance by 15 nucleotides, as is the case in $\alpha$ - and $\gamma$-coronaviruses (fig. 3 ), decreases efficiency by $\sim 20 \%$, while removal of the stop decreases it by half. 


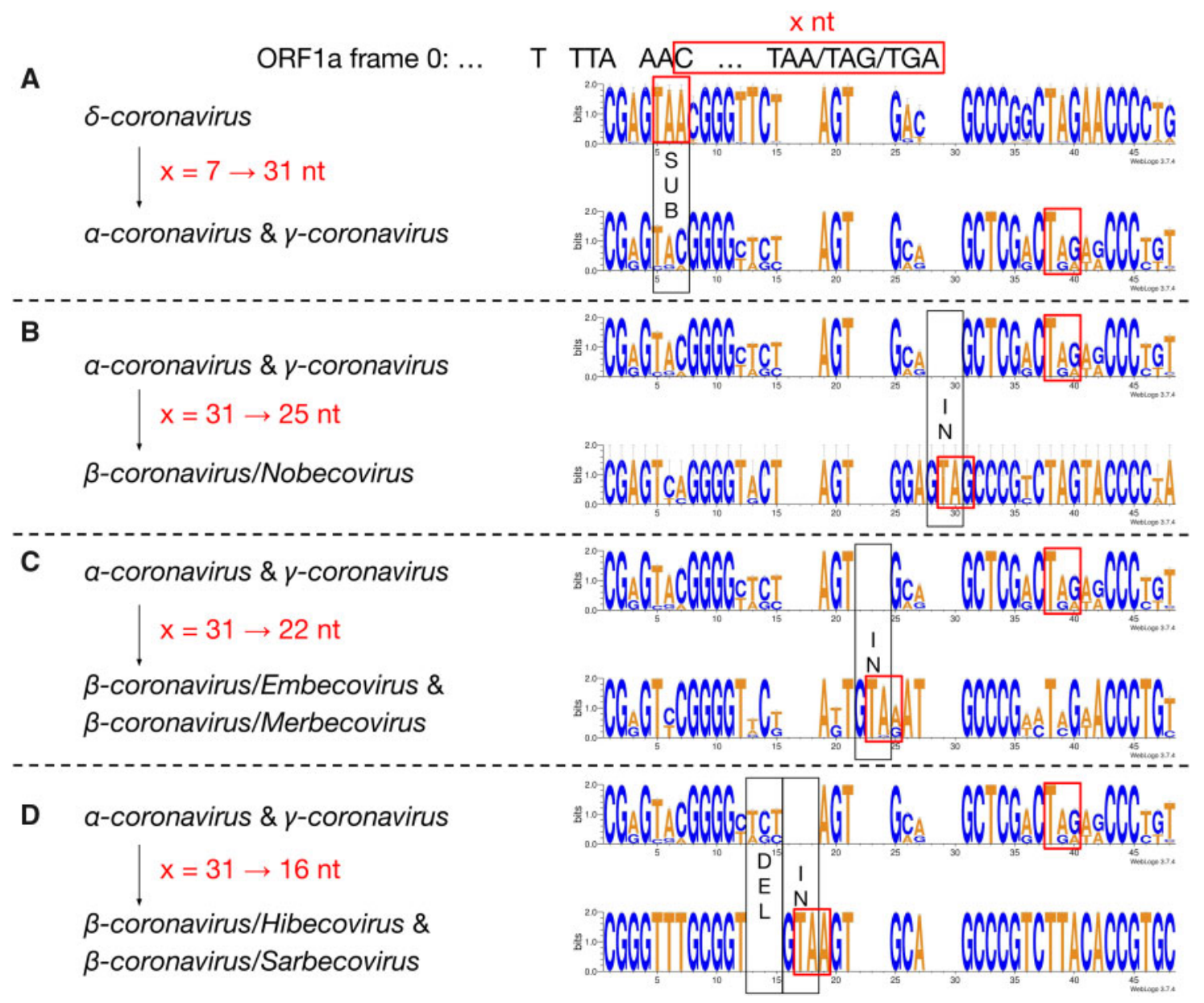

Fig. 3. Nucleotide alignment of the overlap in coronaviruses with $7,31,25,22$, and $16 \mathrm{nt}$. The footprints of substitutions, insertions, and deletions are shown in black boxes, and labeled as "SUB," “IN," and "DEL", respectively. The stop codon of ORF1a in each of the 7, 31, 25, 22, and 16 nt overlap coronaviruses is shown in a red box.

The abundance of SARS-CoV-2 sequencing data allows examining the substitution dynamics in population- and individual-level sequencing data. For population-level analysis, we identified variants in the PFE region from $>1,550,000$ genome sequences available from GISAID (see Materials and Methods section). However, because GISAID contains only assembled genomes, these data do not provide information about individual-level (intrasample) variation. Hence, we performed an additional detailed analysis of $>55,000$ samples generated with the COG-UK (Lythgoe et al. 2021) consortium (see Maier et al. (2021) for analysis details). A summary of results from both analyses is shown in table 1. There is little variation in the PFE region as the fraction of samples containing individual substitutions appears to be small (the two "Count" columns in table 1). Furthermore, the 30 out of 36 substitutions in table 1 are consistent with being a result of RNA editing events from APOBEC (Chen and MacCarthy 2017) or ADAR (Bazak et al. 2014) enzymatic complexes. The remaining six substitutions (all transitions) are predominantly located in the loop regions of the predicted PFE secondary structure (Huston et al. 2021) and thus likely have minimal effect on the secondary structure.
Through a comparative analysis of GISAID sequences, we found that several codons with non-negligible levels of variation (table 2) were subject to purifying selection: RdRp: 1 (A13,443>C/G), RdRp: 31 (13,532 A>G/C), RdRp: 32 (13,535 $C>T)$. This is consistent with a strong degree of functional constraint. Interestingly, this analysis also identified a single codon: RdRp: T26I (13,516 T>C), which has been subject to pervasive positive selection since early 2021. Most of the sequences with this substitution are in the B.1.1.7 and B.1.177.77 lineages (this is a consensus majority mutation in B.1.177.77 and B.1.614 lineages). RdRp: T26l is present at low frequencies in many viral lineages but is increasing in prevalence in recent months $(0.5-1.0 \%$ global prevalence in recent samples). Functional significance, if any, for this substitution has not been reported.

Our results provide an alternative way to assess exceptional conservation of the PFE using publicly available sequence data highlighting the fact that the entire PFE region appears to be under strong purifying selection. These patterns are similar to observations obtained from deep mutational scanning where any alteration at the majority of PFE region sites have deleterious effects on the frameshift efficiency (e.g., Carmody et al. 2021). 
Table 1. Allelic Variants within the PFE Region are Called from Complete GISAID Genomes (population) and COG-UK (individual) Data.

\begin{tabular}{|c|c|c|c|c|c|c|c|c|c|}
\hline \multirow[t]{2}{*}{ Site } & \multirow[t]{2}{*}{$\mathbf{H}$} & \multirow[t]{2}{*}{ B } & \multirow[t]{2}{*}{ Reference } & \multicolumn{2}{|c|}{ Population } & \multicolumn{4}{|c|}{ Individual } \\
\hline & & & & Alternate & Count $^{c}$ & Alternate & Min AF & $\operatorname{Max} \mathrm{AF}$ & Count $^{d}$ \\
\hline $13,425^{\mathrm{a}}$ & & & $\mathrm{C}$ & $T$ & 1,812 & - & - & - & - \\
\hline $13,429^{\mathrm{a}}$ & & & $\mathrm{C}$ & $\mathbf{T}$ & 460 & - & - & - & - \\
\hline $13,430^{\mathrm{a}}$ & & & C & $T$ & 169 & - & - & - & - \\
\hline $13,431^{a}$ & & & C & $T$ & 517 & - & - & - & - \\
\hline $13,432^{b}$ & & & A & G & 110 & - & - & - & - \\
\hline $13,434^{\mathrm{a}}$ & & & G & A & 213 & - & - & - & - \\
\hline $13,43^{\mathrm{a}}$ & & & C & T/A & $1,328 / 120$ & $T$ & 0.116 & 0.971 & 14 \\
\hline $13,437^{b}$ & & & $\mathrm{~T}$ & $C$ & 195 & C & 0.985 & 0.988 & 5 \\
\hline 13,440 & $\mathbf{S}$ & $S$ & G & A & 116 & - & - & - & - \\
\hline $13,443^{b} \downarrow$ & & & A & $\mathrm{G} / \mathrm{T}$ & $134 / 22$ & - & - & - & - \\
\hline $13,445^{\mathrm{a}}$ & & & $\mathrm{C}$ & $\mathbf{T}$ & 680 & $\mathbf{T}$ & 0.068 & 0.970 & 25 \\
\hline $13,447^{a}$ & & & G & A & 16 & - & - & - & - \\
\hline $13,451^{a}$ & & & C & $T$ & 393 & $\mathrm{~T}$ & 0.941 & 0.977 & 19 \\
\hline $13,457^{a}$ & & & C & $\mathbf{T}$ & 3,663 & $T$ & 0.052 & 0.963 & 19 \\
\hline $13,458^{a}$ & & & G & - & - & A & 0.069 & 0.970 & 6 \\
\hline 13,458 & $\mathbf{L}$ & $\mathbf{L}$ & G & $T$ & 1,220 & $T$ & 0.080 & 0.976 & 6 \\
\hline $13,481^{b}$ & & & A & G & 9 & - & - & - & - \\
\hline $13,486^{a}$ & & & C & $T$ & 1,656 & $\mathbf{T}$ & 0.055 & 0.965 & 7 \\
\hline $13,487^{b}$ & & & A & G & 151 & G & 0.901 & 0.949 & 12 \\
\hline $13,497^{b}$ & & & A & G & 434 & - & - & - & - \\
\hline $13,498^{a}$ & & & $\mathrm{C}$ & $\mathbf{T}$ & 189 & - & - & - & - \\
\hline $13,500^{\mathrm{a}}$ & & & $\mathrm{C}$ & $T$ & 243 & - & - & - & - \\
\hline 13,504 & & $\mathbf{S}$ & G & $\mathbf{T}$ & 102 & - & - & - & - \\
\hline $13,505^{\mathrm{a}}$ & & & C & $\mathbf{T}$ & 314 & $\mathbf{T}$ & 0.887 & 0.917 & 5 \\
\hline 13,511 & & $S$ & A & $\mathrm{T} / \mathrm{G} / \mathrm{C}$ & $121 / 58 / 11$ & - & - & - & - \\
\hline 13,512 & & $S$ & G & $\mathrm{T}$ & 114 & - & - & - & - \\
\hline $13,513^{\mathrm{a}}$ & & & G & A & 342 & - & - & - & - \\
\hline $13,514^{\mathrm{a}}$ & & & $\mathrm{C}$ & $T$ & 495 & $T$ & 0.065 & 0.889 & 6 \\
\hline $13,516^{\mathrm{a}} \uparrow$ & & & C & $T$ & 4,272 & $T$ & 0.101 & 0.840 & 49 \\
\hline 13,525 & $\mathbf{S}$ & $S$ & A & C & 104 & - & - & - & - \\
\hline $13,526^{b}$ & & & $T$ & $\mathrm{C}$ & 117 & - & - & - & - \\
\hline $13,532^{\mathrm{b}} \downarrow$ & & & A & G & 742 & - & - & - & - \\
\hline $13,535^{\mathrm{a}} \downarrow$ & & & $\mathrm{C}$ & $\mathbf{T}$ & 11,942 & $\mathbf{T}$ & 0.215 & 0.841 & 23 \\
\hline $13,541^{b}$ & & & $\mathbf{T}$ & $\mathrm{C}$ & 26 & - & - & - & - \\
\hline $13,547^{\mathrm{a}}$ & & & C & $\mathrm{T}$ & 675 & $\mathbf{T}$ & 0.067 & 0.898 & 8 \\
\hline $13,550^{a}$ & & & C & $\mathbf{T}$ & 2,346 & $T$ & 0.878 & 0.921 & 11 \\
\hline
\end{tabular}

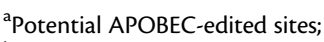

${ }^{\mathrm{b}}$ Potential ADAR-edited sites.

Site numbering is in 0-based coordinates.

'Out of $1,525,442$ complete genome.

${ }^{d}$ Out of 55,163 individual samples. Locations of substitutions in a stem (S) or a loop (L) are based on structures predicted by Huston et al. (H) and Bhatt et al. (B). $\downarrow$ and $\uparrow$

highlight sites showing signatures of negative and positive selection, respectively (see table 2).

Table 2. Sites with Selection Signatures Identified using a Fixed Effects Likelihood Method on Internal Branches using SARS-CoV-2 Phylogeny Built from GISAID Sequences (FEL; [Kosakovsky Pond and Frost 2005]) $\alpha$ : synonymous substitution rate (maximum likelihood estimate, MLE), $\beta$ : non-synonymous substitution rate (MLE), $\omega: \beta / \alpha$.

\begin{tabular}{lccccc}
\hline Codon & Nucleotide & $\alpha$ & $\beta$ & $\omega$ & LRT P value \\
\hline 1 & 13,443 & 0 & 0 & 4.286 & 0.002 \\
31 & 13,352 & 7.040 & 0 & 0 & 0.015 \\
26 & 13,516 & 0 & 4.722 & $\infty$ & 0.004 \\
32 & 13,535 & 5.205 & 0 & 0 & 0.035 \\
\hline
\end{tabular}

\section{Materials and Methods}

\section{Coronavirus Entries Retrieval and Filter}

The 35,152 coronaviral entries in the NCBI taxonomy database were sorted by length, and only those longer than
14,945 nt were kept, leaving a total of 4,939 genomes. The slippery site and following overlap sequences were manually inspected, in case the slippery site was incorrectly annotated. We further filtered out those entries if they contained no annotation information, or had gapped sequences in the overlap. 4,904 coronavirus entries were selected using this approach (supplementary table S1, Supplementary Material online).

\section{Amino Acid Alignment and Nucleotide Alignment of} the Overlap Region

For all $\delta$-coronavirus entries in supplementary table $\mathrm{S} 1$, Supplementary Material online, the first 13 amino acids of ORF1 $b$ were taken to generate a consensus sequence using Weblogo (Crooks et al. 2004). The same was done to $\alpha$-coronavirus and $\gamma$-coronavirus. Within $\beta$-coronavirus, for Nobecovirus, Embecovirus, and Merbecovirus, the first 14 
amino acids were used to build the consensus; for Hibecovirus and Sarbecovirus, the first 13 amino acids were used. In terms of the nucleotide sequence alignments, for each genus/subgenus, the nucleotide sequences used to generate the amino acids mentioned above were taken to make the nucleotide consensus sequence using Weblogo.

\section{Processing of GISAID Data}

Each genome was subjected to codon-aware alignment with the NCBI reference genome (accession number NC_045512) and then subdivided into ten regions based on CDS features: ORF1a (including nsp10), ORF1b (starting with nsp12), S, ORF3a, E, M, ORF6, ORF7a, ORF8, $N$, and ORF10. For each region, we scanned and discarded sequences containing too many ambiguous nucleotides to remove data with possible sequencing errors. Thresholds were $0.5 \%$ for the $S$ gene, $0.1 \%$ for ORF1a and ORF1b genes, and 1\% for all other genes. We mapped individual sequences to the NCBI reference genome (NC_045512) using a codon-aware extension to the SmithWaterman algorithm implemented in the BioExt package (Pond et al. 2005; Gianella et al. 2011) and translated mapped sequences to amino-acids. Codon sequences were next mapped onto the amino-acid alignment. Variants were called directly. Selection analyses were performed using the protocols used previously (Faria et al. 2021; Tegally et al. 2021) based on the FEL analysis (Kosakovsky Pond and Frost 2005) within the HyPhy package (Kosakovsky Pond et al. 2019).

\section{Supplementary Material}

Supplementary data are available at Molecular Biology and Evolution online.

\section{Acknowledgments}

This work is funded by NIH Grants U41 HG006620, R01 Al134384 (NIH/NIAID), and NSF ABI Grant 1661497 and 2027196 (NSF/DBI, BIO). The funders had no role in study design, data collection and analysis, decision to publish, or preparation of the manuscript.

\section{References}

Baranov PV, Henderson CM, Anderson CB, Gesteland RF, Atkins JF, Howard MT. 2005. Programmed ribosomal frameshifting in decoding the SARS-CoV genome. Virology 332(2):498-510.

Bazak L, Haviv A, Barak M, Jacob-Hirsch J, Deng P, Zhang R, Isaacs F), Rechavi G, Li JB, Eisenberg E, et al. 2014. A-to-I RNA editing occurs at over a hundred million genomic sites, located in a majority of human genes. Genome Res. 24(3):365-376.

Bhatt PR, Scaiola A, Loughran G, Leibundgut M, Kratzel A, Meurs R, Dreos R, O'Connor KM, McMillan A, Bode JW, et al. 2021. Structural basis of ribosomal frameshifting during translation of the SARS-CoV2 RNA genome. Science 372(6548):1306-1313.

Brierley I. 1995. Ribosomal frameshifting on viral RNAs. J Gen Virol. 76 ( Pt 8):1885-1892.

Carmody PJ, Zimmer MH, Kuntz CP, Harrington HR, Duckworth KE, Penn WD, Mukhopadhyay S, Miller TF, Schlebach JP. 2021. Coordination of -1 programmed ribosomal frameshifting by transcript and nascent chain features revealed by deep mutational scanning. bioRxiv [Preprint]. 2021 Mar 25:2021.03.25.437046. doi: 10.1101/2021.03.11.435011v1.
Chen J, MacCarthy T. 2017. The preferred nucleotide contexts of the AID/APOBEC cytidine deaminases have differential effects when mutating retrotransposon and virus sequences compared to host genes. PLoS Comput Biol. 13(3):e1005471.

Chung W-Y, Wadhawan S, Szklarczyk R, Pond SK, Nekrutenko A. 2007. A first look at ARFome: dual-coding genes in mammalian genomes. PLoS Comput Biol. 3(5):e91.

Coronaviridae Study Group of the International Committee on Taxonomy of Viruses. 2020. The species severe acute respiratory syndrome-related coronavirus: classifying 2019-nCoV and naming it SARS-CoV-2. Nat Microbiol. 5:536-544.

Crooks GE, Hon G, Chandonia J-M, Brenner SE. 2004. WebLogo: a sequence logo generator. Genome Res. 14(6):1188-1190.

de Groot RJ, Baker SC, Baric R, Enjuanes L, Gorbalenya AE, Holmes KV, Perlman S, Poon L, Rottier PJM, Talbot PJ, et al. 2012. Family coronaviridae. In: King AMQ, Lefkowitz E, Adams MJ, Carstens EB, editors. Virus taxonomy: ninth report of the International Committee on Taxonomy of Viruses. Amsterdam: Elsevier. p. 806-828.

Faria NR, Mellan TA, Whittaker C, Claro IM, da S, Candido D, Mishra S, Crispim MAE, Sales FCS, Hawryluk I, McCrone JT, et al. 2021. Genomics and epidemiology of the P.1 SARS-CoV-2 lineage in Manaus, Brazil. Science 372(6544):815-821.

Gianella S, Delport W, Pacold ME, Young JA, Choi JY, Little SJ, Richman DD, Kosakovsky Pond SL, Smith DM. 2011. Detection of minority resistance during early HIV-1 infection: natural variation and spurious detection rather than transmission and evolution of multiple viral variants. J Virol. 85(16):8359-8367.

Huston NC, Wan H, Strine MS, de Cesaris Araujo Tavares R, Wilen CB, Pyle AM. 2021. Comprehensive in vivo secondary structure of the SARS-CoV-2 genome reveals novel regulatory motifs and mechanisms. Mol Cell. 81(3):584-598.e5.

Iserman C, Roden CA, Boerneke MA, Sealfon RSG, McLaughlin GA, Jungreis I, Fritch EJ, Hou YJ, Ekena J, Weidmann CA, et al. 2020. Genomic RNA elements drive phase separation of the SARS-CoV2 nucleocapsid. Mol Cell. 80(6):1078-1091.e6.

Kelly JA, Olson AN, Neupane K, Munshi S, San Emeterio J, Pollack L, Woodside MT, Dinman JD. 2020. Structural and functional conservation of the programmed -1 ribosomal frameshift signal of SARS coronavirus 2 (SARS-CoV-2). J. Biol. Chem. 295(31):10741-10748.

Kosakovsky Pond SL, Frost SDW. 2005. Not so different after all: a comparison of methods for detecting amino acid sites under selection. Mol Biol Evol. 22(5):1208-1222.

Kosakovsky Pond SL, Poon AFY, Velazquez R, Weaver S, Hepler NL, Murrell B, Shank SD, Magalis BR, Bouvier D, Nekrutenko A, et al. 2020. HyPhy 2.5-a customizable platform for evolutionary hypothesis testing using phylogenies. Mol Biol Evol. 37(1):295-299.

Lau SKP, Woo PCY, Yip CCY, Fan RYY, Huang Y, Wang M, Guo R, Lam CSF, Tsang AKL, Lai KKY, et al. 2012. Isolation and characterization of a novel Betacoronavirus subgroup A coronavirus, rabbit coronavirus HKU14, from domestic rabbits. J. Virol. 86(10):5481-5496.

Lythgoe KA, Hall M, Ferretti L, de Cesare M, Maclntyre-Cockett G, Trebes A, Andersson M, Otecko N, Wise EL, Moore N, et al.; on behalf of the Oxford Virus Sequencing Analysis Group (OVSG). 2021. SARS-CoV2 within-host diversity and transmission. Science. 372(6539):eabg0821.

Maier W, Bray S, van den Beek M, Bouvier D, Coraor N, Miladi M, Singh B, De Argila JR, Baker D, Roach N, et al. 2021. Freely accessible ready to use global infrastructure for SARS-CoV-2 monitoring. bioRxiv [Preprint]. 2021 Mar 25:2021.03.25.437046. doi: 10.1101/ 2021.03.25.437046.

Nekrutenko A, Wadhawan S, Goetting-Minesky P, Makova KD. 2005. Oscillating evolution of a mammalian locus with overlapping reading frames: an XLalphas/ALEX relay. PLoS Genet. 1(2):e18.

Plant EP, Rakauskaite R, Taylor DR, Dinman JD. 2010. Achieving a golden mean: mechanisms by which coronaviruses ensure synthesis of the correct stoichiometric ratios of viral proteins. I Virol. 84(9):4330-4340 
Pond SL, Frost SD, Muse SV. 2005. HyPhy: hypothesis testing using phylogenies. Bioinformatics. 21(5):676-679.

Rangan R, Watkins AM, Chacon J, Kretsch R, Kladwang W, Zheludev IN, Townley J, Rynge M, Thain G, Das R. 2021. De novo 3D models of SARS-CoV-2 RNA elements from consensus experimental secondary structures. Nucleic Acids Res. 49(6):3092-3108.

Sola I, Almazán F, Zúñiga S, Enjuanes L. 2015. Continuous and discontinuous RNA synthesis in coronaviruses. Annu Rev Virol. 2(1):265-288.

Szklarczyk R, Heringa J, Pond SK, Nekrutenko A. 2007. Rapid asymmetric evolution of a dual-coding tumor suppressor INK4a/ARF locus contradicts its function. Proc Natl Acad Sci U S A. 104(31):12807-12812.

Tegally $\mathrm{H}$, Wilkinson $\mathrm{E}$, Giovanetti $M$, Iranzadeh A, Fonseca $V$, Giandhari J, Doolabh D, Pillay S, San EJ, Msomi N, et al. 2021. Detection of a
SARS-CoV-2 variant of concern in South Africa. Nature 592(7854):438-443.

Woo PCY, Lau SKP, Lam CSF, Lau CCY, Tsang AKL, Lau JHN, Bai R, Teng JLL, Tsang CCC, Wang M, et al. 2012. Discovery of seven novel Mammalian and avian coronaviruses in the genus deltacoronavirus supports bat coronaviruses as the gene source of alphacoronavirus and betacoronavirus and avian coronaviruses as the gene source of gammacoronavirus and deltacoronavirus. J Virol. 86(7):3995-4008.

Ziv O, Price J, Shalamova L, Kamenova T, Goodfellow I, Weber F, Miska EA. 2020. The short- and long-range RNA-RNA interactome of SARS-CoV-2. Mol Cell. 80(6):1067-1077.e5. 